NOTA CIENTÍFICA

\title{
Primer registro de Heterocucumis godeffroyi (Semper, 1868) (Echinodermata: Holothuroidea) en el mar peruano
}

\author{
First record of Heterocucumis godeffroyi (Semper, 1868) (Echinodermata: \\ Holothuroidea) from Peruvian sea
}

\author{
Francisco Alonso Solís-Marín ${ }^{1}$, Yuri Hooker ${ }^{2}$, Andrea Alejandra Caballero Ochoa ${ }^{3}$ \\ y Alfredo Laguarda-Figueras ${ }^{1}$
}

1 Laboratorio de Sistemática y Ecología de Equinodermos, Instituto de Ciencias del Mar y Limnología (ICML), Universidad Nacional Autónoma de México (UNAM). Apdo. Post. 70-305, México, D. F. 04510, México.

E-mail Francisco Solís-Marín: fasolis@cmarl.unam.mx

2 Laboratorio de Biología Marina, Departamento de Ciencias Biológicas y Fisiológicas, Universidad Peruana Cayetano Heredia. Av. Honorio Delgado 430, Urb. Ingeniería, S.M.P. Lima, Perú.

3 Posgrado en Ciencias del Mar y Limnología, Instituto de Ciencias del Mar y Limnología (ICML), Universidad Nacional Autónoma de México (UNAM).

Presentado: $\quad 16 / 10 / 2012$ Aceptado: $\quad$ 05/12/2012 Publicado online: $15 / 01 / 2013$

\section{Resumen}

Se registra por primera vez para el Perú la especie de pepino de mar Heterocucumis godeffroyi (Semper, 1868) (Echinodermata: Holothuroidea). La especie fue recolectada a $19 \mathrm{~m}$ de profundidad en Pucusana, departamento de Lima. Este registro representa el límite más norteño de distribución de la especie reconocido hasta el momento. Material de referencia se encuentra depositado en la Colección de Zoología Acuática, del Laboratorio de Biología Marina, Departamento de Ciencias Biológicas y Fisiológicas, Universidad Peruana Cayetano Heredia, Lima, Perú.

Palabras clave: Primer Registro; pepino de mar; biodiversidad marina.

\section{Abstract}

This is the first record of Heterocucumis godeffroyi (Semper, 1868) (Echinodermata: Holothuroidea) for Peruvian waters. The species was collected at19 m deep, in Pucusana, off Lima Departamento. This record represents the northern most distribution limit knowing to the present. Reference material has been deposited at the Colección de Zoología Acuática, Laboratorio de Biología Marina, Departamento de Ciencias Biológicas y Fisiológicas, at Universidad Peruana Cayetano Heredia, Lima, Perú.

Keywords: First record; sea cucumber, marine biodiversity.

\section{Introducción}

Existen pocos estudios taxonómicos sobre los pepinos de mar peruanos. Los holoturoideos de Perú están representados por aproximadamente 74 especies (Solís-Marín et al. 2012; Hooker et al. 2013) la gran mayoría de estos corresponden a especies de zonas someras $(>200 \mathrm{~m})$.

H. L. Clark (1910) registró algunas especies de pepinos de mar recolectadas en mar territorial peruano, o que posiblemente se podrían encontrar en la misma zona: Stichopus fuscus (=Isostichopus fuscus), Holothuria chilensis, Phyllophorus peruvianus (=Pattalus mollis), Cucumaria leonina (=Pseudocnus dubiosus), Colochirus peruanus (=Trachythyone peruana) y Thyone gibber (=Neothyone gibber).

Posteriormente, Deichmann $(1941,1958)$ reportó 14 especies de holoturias recolectadas durante las expediciones Allan Hancock en el Pacífico americano (Velero III y IV) entre 1932 y 1938, y registró algunas para el Perú: Athyonidium chilensis, Cucumaria californica (=Pseudocnus californicus), Cucumaria dubiosa (=Pseudocnus dubiosus), Euthyonidium ovulum (=Afrocucumis ovulum), Neothyone gibber, Neothyone gibosa, Neothyone panamensis, Pattalus mollis, Pentamera chiloensis, Psolidium planum, Anaperus peruviana, Pentacta peruana (=Trachythyone peruana), Ludwigothuria kefersteini (=Holothuria kefersteini) y Selenkothuria theeli (=Holothuria theeli).

Hooker et al. (2005) registraron 13 especies de holoturoideos en el inventario de equinodermos de las islas Lobos de Afuera (Lambayeque, Perú), de los cuales seis fueron nuevos registros para el Perú: Psolidium dorsipes, Cucumaria flamma, Pseudocnus californicus, Holothuria sp., Chiridota sp. y Actinopyga sp., y mencionan que los ejemplares no identificados de Holothuria sp. y Actinopyga sp. no correspondieron a ninguno de los holoturoideos conocidos en el Pacífico Oriental.

Prieto-Ríos (2011) reportó 22 especies de holoturoideos habitantes del mar del Perú, distribuidas en tres órdenes, ocho familias y trece géneros, aportando ocho nuevos registros para el mar del Perú: Pentamera chierchia, Neocucumis veleronis, Holothuria (Cystipus) inhabilis, Holothuria (Selenkothuria) lubrica, Holothuria (Semperothuria) imitans, Holothuria (Vaneyothuria) zacae, Molpadia intermedia y Caudina californica.

Recientemente Solís-Marín et al. (2012) reportaron la existencia de Enipniastes eximia (una especie de holoturia pelágica) frente a las aguas del mar peruano.

H. L. Clark (1910) fue el primero en sugerir la existencia de Cucumaria godeffroyi (=Heterocucumis godeffroyi) en las aguas del Perú, sin embargo, nunca observó y/o analizó ningún ejemplar recolectado en aguas peruanas. Es hasta ahora que se registra formalmente, y con la existencia de un reporte físico a $H$. godeffroyi (Semper, 1868) para el Perú. Este registro representa el límite de distribución geográfica más norteño de la especie reconocido hasta el momento. Material de referencia se encuentra depositado en la Colección de Zoología Acuática (CZA), del Laboratorio de Biología Marina, Departamento de Ciencias Biológicas y Fisiológicas, Universidad Peruana Cayetano Heredia, Lima, Perú. 


\section{Sistemática}

Phylum Echinodermata Klein, 1734 (ex Bruguiére, 1789)

Clase Holothuroidea BlainVille, 1834

Orden Dendrochirotida Grube, 1840

FAMILIA CUCUMARIIDAE LUDWIG, 1894

\section{Heterocucumis godeffroyi (Semper, 1868)}

(Figuras 1-3)

Cucumaria godeffroyi Semper, 1868: 53, pl. 15, figs. 12, 14; H.L. Clark, 1910: 352-353; Deichmann, 1941: 83-84; 1947: 5; Pawson, 1969: 131-133, fig. 1; Lambert, 2009: 869, text-fig.

Heterocucumis godeffroyi.- O'Loughlin, 2002: 316-317; O'Loughlin et al., 2009: 14 (lista).

Material examinado: Un ejemplar, CZA-381, Bahía de Pucusana, Lima, Perú, (12²8'14,73”S, 76²8’2,52”W), a 50 kilómetros de Lima, 25 de marzo 2012, 19 m, bajo rocas, recolector: Yuri Hooker.

Descripción. Ejemplar adulto de tamaño grande $(33,80 \mathrm{~mm})$ (Fig. 1A). En alcohol el material examinado es amarillento con café obscuro en piel y tentáculos. Cuerpo contraído de forma más o menos cilíndrica. Podios en cinco series dispuestos en los ambulacros, cada serie con dos filas (Fig. 1B); da la apariencia de tener numerosas filas, efecto observado debido a la contracción del ejemplar. Los pies ambulacrales de ejemplares más pequeños generalmente ausentes en el dorso (Pawson 1969). Diez tentáculos dendríticos, de tamaño desigual (de 3,15 a 3,35 mm de largo en el ejemplar contraído) (Fig. 1C, D). El anillo calcáreo es simple, sin proyecciones. Las espículas de la pared del cuerpo son placas perforadas $(4-14$ orificios, en su mayoría de $6-$ 10), pequeñas, de diversos tamaños (Fig. 2A, C), con bordes y superficie espinosa; algunas con proyecciones marginales. Pies ambulacrales con barrotes de soporte curvados, en algunos casos, la parte central con una corona de espinas o pequeñas perforaciones (Fig. 2B). Las espículas de los tentáculos son barrotes lisos, perforados (Fig. 2D). Introverso con barrotes lisos y perforados (Fig. 2E), más elaborados que los de los tentáculos.

Holotipo: Museo de Hamburgo, Alemania (Deichmann 1941).

Localidad tipo: Iquique, Chile (Semper 1868).

Referencias de identificación: Pawson, 1969, Fig. 1; Lambert, 2009, text-fig. pág. 869.

Distribución geográfica: Originalmente descrita para la costa de Iquique, Chile (Semper 1868). Es muy probable que se distribuya a todo lo largo de la costa chilena (Pawson 1969). Deichmann $(1941,1947)$ menciona que su intervalo de distribución va de "la costa oeste de Sud América" entre $20^{\circ}$ y $40^{\circ}$ de latitud $S$, probablemente porque los dos ejemplares reportados por ella en su trabajo de 1947 fueron localizados en las vecindades de la Isla Chiloé (Pawson 1969).
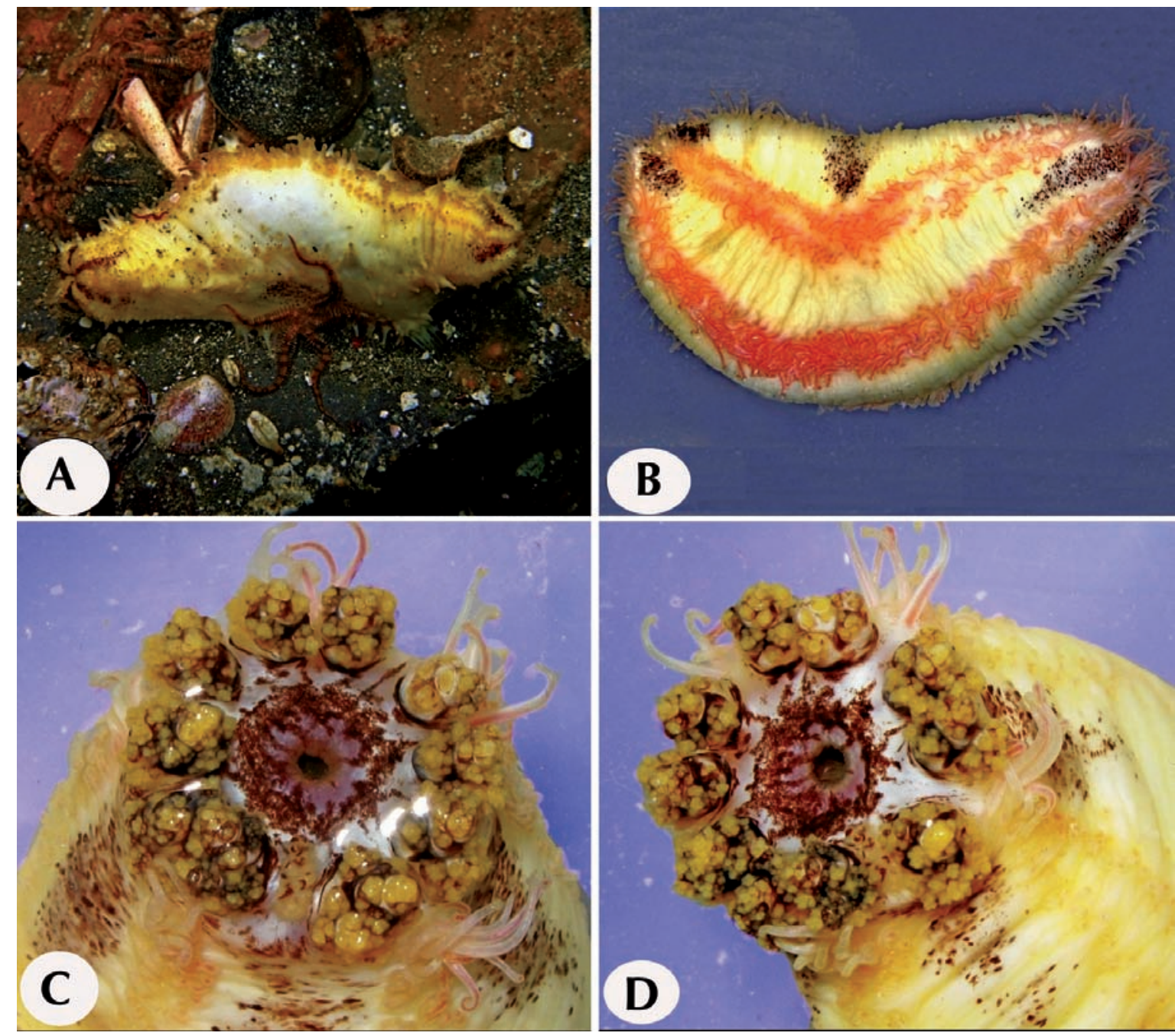

Figura 1. Heterocucumis godeffroyi (Semper, 1868). A. Espécimen in situ, B. espécimen vivo mostrando los pies ambulacrales extendidos, C y D. Detalles de la zona anterior del cuerpo mostrando los tentáculos dendríticos retraídos. Fotos: Yuri Hooker. 


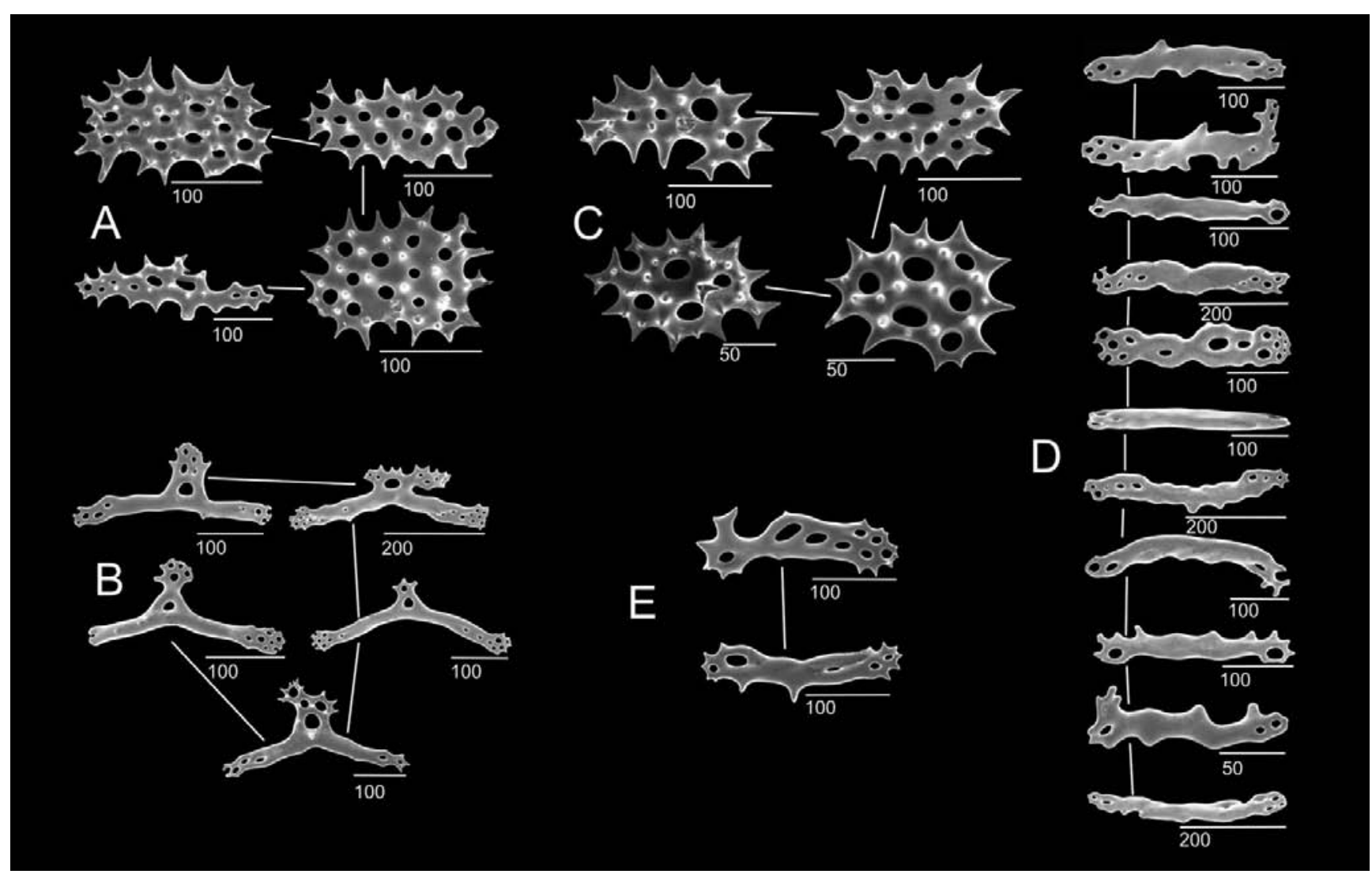

Figura 2. Heterocucumis godeffroyi. A: placas perforadas del ambulacro; B: barrotes del ambulacro; C: placas perforadas del interambulacro; D: barrotes de los tentáculos; E: barrotes del introverso. Escalas en micras.

Pawson (1969) extendió el límite sur de la distribución de la especie hasta los 41 $50^{\prime} 30^{\prime} \mathrm{S}$.

O’Loughlin (2002) amplió el rango de distribución geográfica de esta especie desde la región Magallánica a la región de las Islas Subantárticas (Kerguelen, $49^{\circ} \mathrm{S}, 70^{\circ} \mathrm{E}$; Isla Heard $51-53^{\circ} \mathrm{S}$, $73-76^{\circ} \mathrm{E}$ ) (Fig. 3), estos registros requieren verificación dada la lejanía de las islas subantárticas con respecto a la zona de distribución geográfica conocida de la especie en América del Sur. En este trabajo se reporta por primera vez, el límite más norteño de la especie en aguas peruanas (Fig. 3).

Distribución batimétrica: De 0 a 60 m (Pawson 1969). De 4 a 379 m (O'Loughlin 2002; Lambert 2009).

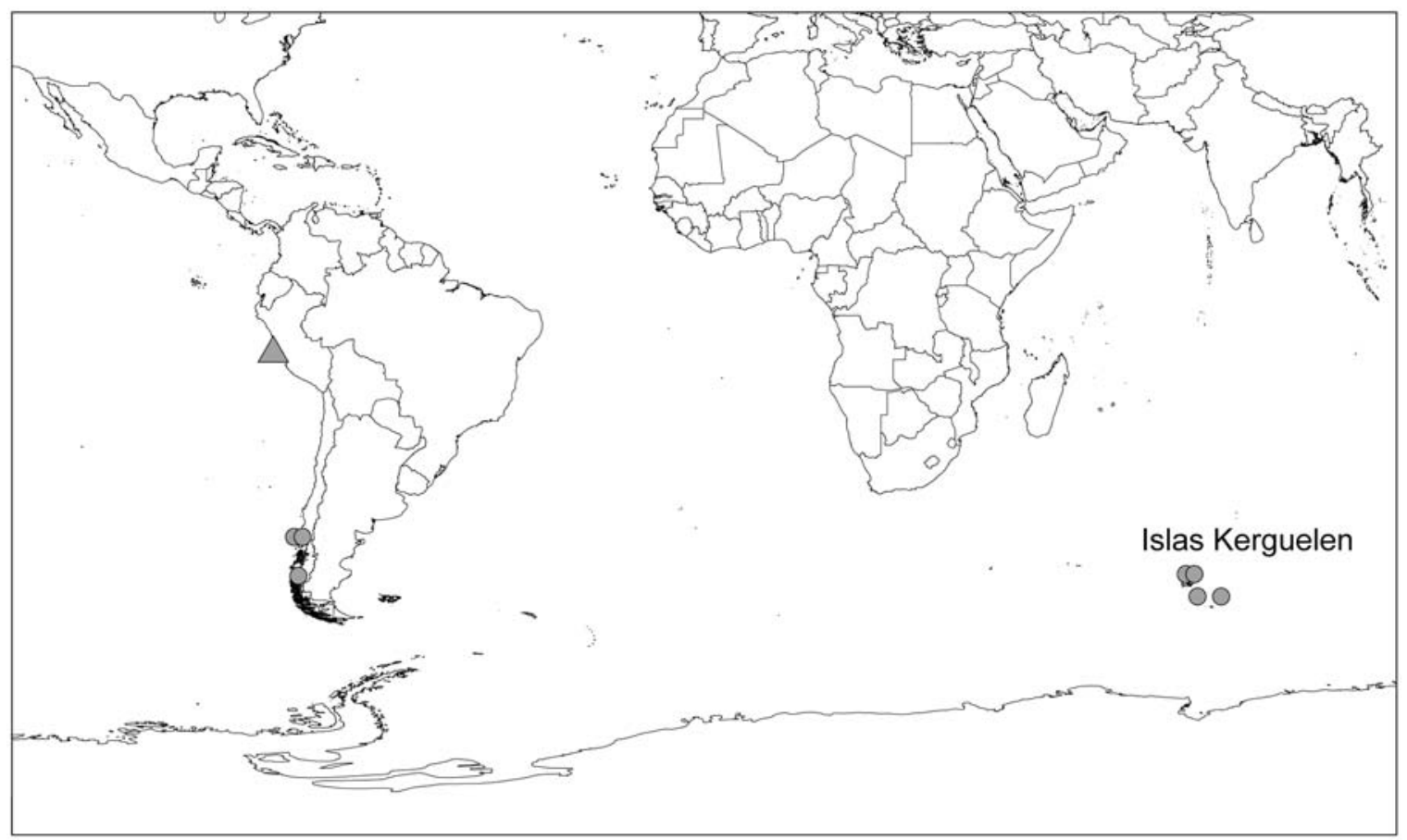

Figura 3. Mapa de distribución de Heterocucumis godeffroyi (Semper, 1868) en el Pacífico Sur oriental ( $\boldsymbol{\Delta}$ nuevo registro). 
Hábitat: habita sustratos de arena gruesa y conchuela. También se le puede localizar bajo rocas y asociado a tubos de poliquetos (Pawson 1969; Lambert 2009). Nuestro espécimen fue recolectado debajo de una roca, adherida a ella (Fig. 1A).

Color: El color en vivo observado es blanco amarillento con tentáculos amarillo claro, la superficie oral y tentáculos son moteados con manchas marrón obscuro. Espacios interambulacrales de los extremos anterior y posterior del cuerpo, así como la zona medio-dorsal con pequeñas puntos de color marrón oscuro (Fig. 1). Pawson (1969) menciona que los individuos juveniles presentan diferencias en color y tamaño de tentáculos ventrales

Observaciones: La mayoría de los registros de $H$. godeffroyi se han presentado en aguas frías y templadas de la zona del Pacífico Sur Oriental, que según Brusca y Wallerstein (1979), Jaramillo (1982), Hendrickx (1992) y Lancellotti y Vázquez (1999), corresponde a las provincias biogeográficas Magallánica y Templada-Transicional; este nuevo registro muestra la presencia de la especie en aguas más cálidas-templadas (Provincia PerúChile) que evidencia una transición hacia la zona tropical (Provincia Panámica). Este resultado coincide con lo propuesto por Deichmann (1941, 1959), Ekman (1953), Caso (1978, 1979) y Maluf (1988) que mencionan a Perú (Isla Lobos de Afuera) como el límite Sur de la distribución de un número importante de especies de equinodermos tropicales.

\section{Agradecimientos}

A Alicia Durán González, Técnico Académico de la Colección Nacional de Equinodermos de México (ICML, UNAM) por el trabajo técnico desarrollado para la realización de esta publicación. A Berenit Mendoza Garfias quien tomó las fotografías de microscopía electrónica de barrido (Laboratorio de Microscopía Electrónica del Instituto de Biología, UNAM) que ilustran el presente trabajo.

\section{Literatura citada}

Brusca R.C. \& B.R. Wallerstein. 1979. Zoogeographic patterns of idoteid isopods in the northeast Pacific, with a review of shallow water zoogeography of the area. Bull. Bio. Soc. of Washington. (3): 67-105.

Caso M.E. 1978. Los equinodermos del Pacífico de México. Parte primera: Ordenes Cidaroidea y Aulodonta. Parte segunda - Ordenes Stiridonta y Camarodonta. Centro Cienc. del Mar y Limnol. UNAM, Publ. Esp. 1:1-244.

Caso M.E. 1979. Los Equinodermos de la Bahía de Mazatlán, Sinaloa. Centro Cienc. del Mar y Limnol. Univ. Nal. Autón. México. 6(1):167-368.

Clark H.L. 1910. The Echinoderms of Peru. Bulletin of the Museum of Comparative Zoology at Harvard University, 52(17): 321-358.

Deichmann E. 1941. The holothuroidea collected by the Velero III during the years 1932 to 1938 . Part I. Dendrochirota. The University of Southern California Publications, Allan Hancock Pacific Expeditions 8(3): 61-195.
Deichmann E. 1947. Shallow water Holothurians from Cabo de Hornos and adjacent waters. Anales del Museo Argentino de Ciencias Naturales "Bernardino Rivadavia". Invertebrados Marinos, 42(8): 325-352.

Deichmann E. 1958. The Holothuroidea collected by the Velero III and IV during the years 1932 to 1954. Part. II Aspidochirota. Allan Hancock Pac. Exped., 11(2):253-348.

Deichmann E. 1959. Ekman's Barrier and the Holothurians of the Panama Region. Proc. Int. Congr. Zool., 15: 270-272.

Ekman S. 1953. Zoogeography of the sea. Sidgwick and Jackson Limited. London. 417 pp.

Hendrickx M.E. 1992. Distribution and zoogeographic affinities of decapod crustaceans of the Gulf of California, Mexico. Proc. San Diego Soc. Nat. Hist., 20: 1-11.

Hooker Y, Solís-Marín F.A. \& Llellish M. 2005. Equinodermos de las Islas Lobos de Afuera (Lambayeque, Perú). Revista Peruana de Biología, 12(1):77-82.

Hooker Y, Prieto-Rios E. \& F.A. Solís-Marín. 2013. Echinoderms of Peru. p. 277-299. In. Alvarado-Barrientos, J.J. \& F.A. Solís-Marín. 2012. Echinoderm research and Diversity in Latin America. Springer. 658 pp. ISBN 978-3-64220050-2.

Jaramillo E. 1982. Taxonomy, natural history and zoogeography of sand beach isopods from the coasts of Southern Chile. Stud. Neotrop. Fauna \& Environ., 17(4): 175-194.

Lambert P. 2009. Holothuroidea- Pepinos de mar. pp. 859-880. In. Häussermann V. y G. Fösterra (eds.). 2009. Fauna Marina Bentónica de la Patagonia Chilena. 1a. Ed. Nature in Focus, Chile. 1000 p.

Lancellotti D.A. \& J.A. Vásquez. 1999. Biogeographical patterns of benthic macroinvertebrates in the Southeastern Pacific litoral. Journal of Biogeography. 26 (5): 1001-1006.

Maluf L. 1988.Composition and Distribution of the Central Eastern Pacific Echinoderms. Natural History Museum of Los Angeles County. Technical Reports, No. 2. 242 pp.

O’Loughlin P.M. 2002. Report on selected species of Banzare and Anare Holothuroidea, with reviews of Meseres Ludwig and Heterocucumis Panning (Echinodermata). Memoirs of Museum Victoria, 59(2): 297- 325.

O’Loughlin P.M., Manjón-Cabeza M.E. \& F. Moya Ruiz. 2009. Antarctic holothuroids from the Bellingshausen Sea, with descriptions of new species (Echinodermata: Holothuroidea). Zootaxa, 2016: 1-16.

Pawson D.L. 1969. Holothuroidea from Chile. Report. No. 46 of the Lund University Chile Expedition 1948-1949. Sarsia, 38: 121-145.

Prieto-Rios E. 2010. Taxonomía de Holothuroidea (Echinodermata) del mar del Perú. Tesis Profesional, Facultad de Ciencias Biologicas, Universidad Nacional Mayor de San Marcos, Lima, Perú.

Semper C. 1868. Reisen im Archipel der Philippinen. II. Wissenschaftliche Resultate. Vil. I, Holothurien, 288 pp., 40 pls.

Solís-Marín F.A., Hooker Y \& A Laguarda-Figueras. 2012. Primer registro del pepino de mar nadador Enypniastes eximia Théel, 1882 (Echinodermata: Holothuroidea) en aguas peruanas. Revista Peruana de Biología, 19(1): 95-96. 